\title{
To Stretch the Boundary of Secondary Metabolite Production in Plant Cell-Based Bioprocessing: Anthocyanin as a Case Study
}

\author{
Wei Zhang, ${ }^{1,2 *}$ Chris Franco, ${ }^{1}$ Chris Curtin, ${ }^{1}$ and Simon Conn ${ }^{1}$ \\ ${ }^{1}$ CRC for Bioproducts and Department of Medical Biotechnology, School of Medicine, \\ Flinders Medical Centre, Flinders University, SA 5042, Adelaide, Australia \\ ${ }^{2}$ Marine Bioproducts Engineering Group, Dalian Institute of Chemical Physics, \\ Chinese Academy of Sciences, Dalian 116023, China
}

Received 19 April 2004; revised 27 May 2004; accepted 15 June 2004

\begin{abstract}
Plant cells and tissue cultures hold great promise for controlled production of a myriad of useful secondary metabolites on demand. The current yield and productivity cannot fulfill the commercial goal of a plant cell-based bioprocess for the production of most secondary metabolites. In order to stretch the boundary, recent advances, new directions and opportunities in plant cell-based bioprocessing have been critically examined for the 10 years from 1992 to 2002 . A review of the literature indicated that most of the R\&D work was devoted predominantly to studies at an empirical level. A rational approach to molecular plant cell bioprocessing based on the fundamental understanding of metabolic pathways and their regulations is urgently required to stimulate further advances; however, the strategies and technical framework are still being developed. It is the aim of this review to take a step forward in framing workable strategies and technologies for molecular plant cell-based bioprocessing. Using anthocyanin biosynthesis as a case study, an integrated postgenomic approach has been proposed. This combines the functional analysis of metabolic pathways for biosynthesis of a particular metabolite from profiling of gene expression and protein expression to metabolic profiling. A global correlation not only can thus be established at the three molecular levels, but also places emphasis on the interactions between primary metabolism and secondary metabolism; between competing and/or complimentary pathways; and between biosynthetic and post-biosynthetic events.
\end{abstract}

\section{INTRODUCTION}

Plants are probably the best cell factories on this planet from which more than 100000 low molecular secondary metabolites have been discovered, with the estimated total number in plants exceeding 500000 [1]. Plant metabolites not only are used for food purposes but also serve as an important historical source of medicines. Plant cell-based bioprocessing is the use of the biosynthetic pathways of plant cells/tissues for the production of valuable metabolites and for biotransformation. Despite worldwide effort over 40 years, "potential" is still the word most frequently used to describe this technology as it has met with very limited commercial success $[2,3]$. To advance our knowledge and tools in translating "potential" into "commercial success," the present paper examines the recent advances in this area and intends to establish strategies and the technical framework for a rational molecular bioprocessing approach. To illustrate this approach, we present some data on the functional analysis of metabolic pathways for biosynthesis of anthocyanins: from profiling of gene expression and protein expression to metabolic profiling in Vitis vinifera cell culture as a model system. Emphasis was placed on a global correlation at three molecular levels-gene transcript, enzyme, and metabolite - as well as on the interactions between the biosynthetic pathway and post-biosynthetic events. The latter has been largely overlooked in the past.

Focusing on the fundamental understanding of the complex metabolic pathway and regulation of secondary metabolism in plant cell cultures, we are developing advanced knowledge of biosynthesis as well as postbiosynthesis pathways of anthocyanins from the genetic to the metabolite level $[2,4,5,6,7]$. The metabolic pathways were characterized dynamically using techniques such as precursor feeding, elicitation, metabolic inhibition and analysis of strains, mRNA expression, enzyme activities, and anthocyanin profiles. Information gained on identification, regulation, and manipulation of the limiting and key steps involved in the anthocyanin pathways is leading us to new avenues for a rational bioprocess engineering optimization of anthocyanin production at both the molecular and the cellular level. One of our current aims is to develop an integrated process that rationally combines different enhancement strategies for further productivity increases in anthocyanin production in 


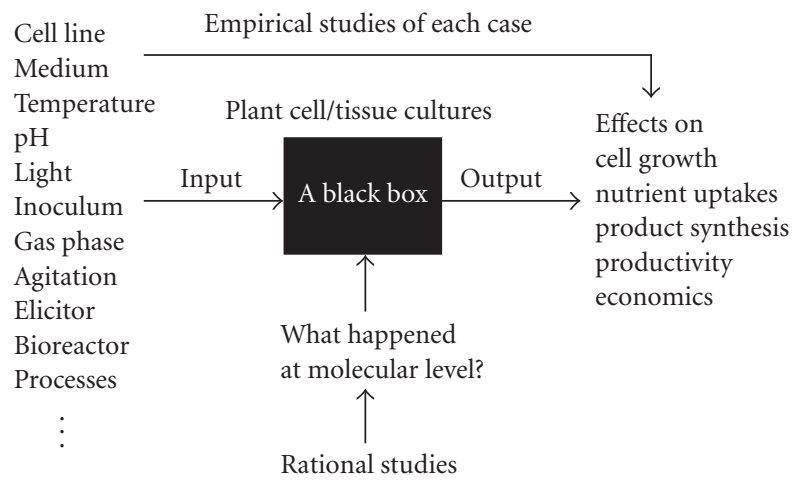

FIGURE 1. Moving from empirical to rational approaches for the understanding of plant cell-based bioprocessing at molecular level (genetic, enzymatic, and metabolic levels) which remains a black box at large.

$V$ vinifera cell cultures [3]. Another interesting strategy is to elucidate the mechanism for anthocyanin transport and storage in plant cells with the aim of manipulating the transport and storage for enhancing anthocyanin production.

\section{RATIONAL APPROACH: EXPECTATION AND REALITY}

Empirical approaches have been employed for the development and optimization of plant cell-based bioprocesses since their onset. The typical feature of the empirical approaches is to optimize the plant cell culture system with regard to its input factors (cell line, medium, culture parameters, bioreactors, process operations, etc) and output factors (cell growth, nutrient uptake, productivity, yield, etc). As illustrated in Figure 1, what occurs at the cellular and molecular levels remains largely unknown, as a "black box."

This "brute-force" empirical approach has to be applied to every plant cell culture process, which is timeconsuming, costly, and often suboptimal [8, 9]. As demonstrated by the limited commercial success to date, There may be little hope in stretching the biosynthetic capability of secondary metabolites in plant cell bioprocesses. Rational approaches, in contrast, which have been proposed since the mid-1980s, are directed to the understanding of the black box with the rapid development of modern genetic technology [10]. With the expectation of a radical improvement in biosynthetic capability through engineering secondary metabolism in plant cells, the major challenge for a rational approach is to obtain information on the identification and regulation of biosynthetic genes and pathways.

To obtain a clear picture of what has been done in the development of rational approaches, a literature survey on plant cell/tissue cultures was carried out from 1992 to 2002 using Biological Abstracts. Although it was not intended to be a thorough survey, its results presented in Figure 2 did reveal some interesting information. The work is considered to be a rational study when it is de- voted to characterizing the pathway genes, enzymes, cell physiology, and metabolite profiling. Otherwise it is considered to be an empirical study. Of the nearly 600 publications during this period, an average of $37 \%$ contributed to the rational approach, leaving the majority still in the empirical study domain (Figure 2). The movement toward a rational understanding is rather consistent, with no increasing trend (Figure 2). Furthermore, most of the rational studies are focused on a small handful of secondary metabolites, such as alkaloids (ajmalicine, vincristine, and vinblastine) and flavonoids (anthocyanins). The question is therefore raised: why has the R\&D effort toward a rational approach in plant cell bioprocesses remained so stagnant given the rapid entry into the postgenomic era during the past 10 years?

\section{RATIONAL APPROACH: CHALLENGES AND POTENTIALS}

The primary task of a rational approach for plant cell bioprocessing is to identify the biosynthetic genes, enzymes, and pathways for a specific secondary metabolite. This is essentially a functional analysis after the genome discovery. Though advocated since the mid-1980s, it is only recently that the large-scale functional analysis of secondary metabolism becomes feasible with the completion of the sequencing of the Arabidopsis and rice genomes [11]. Technically, the paradigm shift from empirical to rational was born during the past decade and is now at the early stages of expected rapid growth in the next decade. With the continuing success of numerous sequencing projects, we have entered a new era of plant cell-based bioprocessing. Firstly, we have at our disposal an enormous resource of genes, for the functional analysis of their roles, protein products, and pathways and biosynthesis of specific classes of secondary metabolites. Secondly, the accumulated information in empirical studies of plant cell bioprocesses will provide important leads/conditions for such a functional analysis. Thirdly, large-scale, global, and dynamic analysis of 


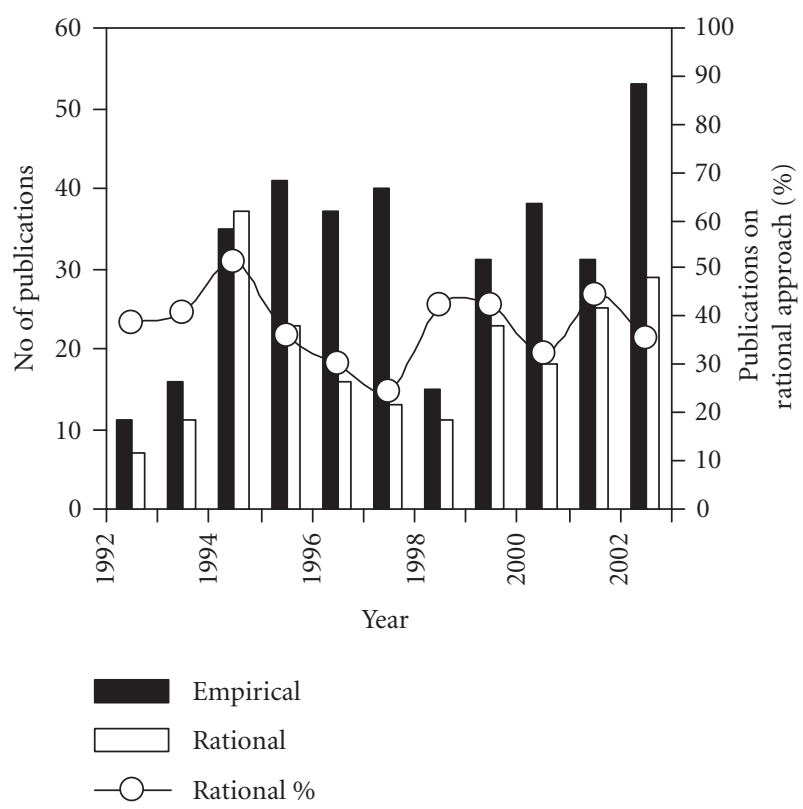

Figure 2. Number of publications on empirical approaches and rational studies of plant cell/tissue cultures, and the percentage of rational studies from 1992 to 2002. The search is based on the Biological Abstracts.

secondary metabolism will become feasible with the rapid advances in post-genomic tools, such as transcriptomics, proteomics, and metabolomics in this new era. This analysis will address the interactions at a dynamic and global level among the primary metabolism, the biosynthetic pathways for secondary metabolism, and the postbiosynthetic events of specific metabolites. As a result, plant cell bioprocess engineers will be empowered with greater capability to stretch the limits of natural biosynthetic pathways of plant cells by mobilizing the genes of interest into transgenic plant cells to perform valuable functions for industry, medicine, and the environment.

Although the future looks brilliant, the strategies and technical framework for a rational approach are still being developed. Below are several major challenges that must be faced:

(1) the extreme complexity of secondary metabolism will demand novel strategies and tools;

(2) the diversity, specificity, and variability of secondary metabolites within and among plant families or species have been the major impediment in the elucidation of many secondary pathways;

(3) regulatory properties in secondary metabolism, such as cell-type-specific localization and transient expression, may complicate the true biosynthetic potential of plant cells;

(4) many plant species have complex genomes; interactions among complex and diverse metabolisms may prevent efficient genetic and metabolic engineering manipulations.

\section{RATIONAL APPROACH: STRATEGIES AND TECHNICAL FRAMEWORK}

Rational manipulation of "linear primary pathways" such as starch biosynthesis is straightforward and has turned out to be successful [12]. However, the biosynthetic pathways of plant secondary metabolites are often extremely complicated as illustrated in Figure 3.

In formulating the strategies for a rational approach, the complexity will need to be considered in terms of linking primary metabolism with secondary metabolism, linking biosynthetic pathways with postbiosynthetic events, as well as linking targeted biosynthetic pathways with competing and/or complimentary pathways (Figure 3). Several strategies have been proposed recently to address the challenges and complexity $[2,11,13]$.

The three main tasks of a rational approach are (i) characterization of all the genes involved, their protein products, and their metabolic products in a given biosynthetic pathway; (ii) characterization of their respective regulatory functions and roles; (iii) manipulation of the biosynthetic pathways for a given application via the engineering of metabolism. Here, we propose a technical framework to implement this approach.

Firstly, the tools of genomics, proteomics, and metabolomics have to be integrated to obtain a global understanding of secondary metabolism at three molecular levels. Any characterization at a single level may fail to be applied for rational bioprocess improvement. Oksman-Caldentey et al [11] have proposed an approach that combines metabolomics and transcriptomics. Using a genome-wide transcript cDNA-AFLP profiling [14] in 


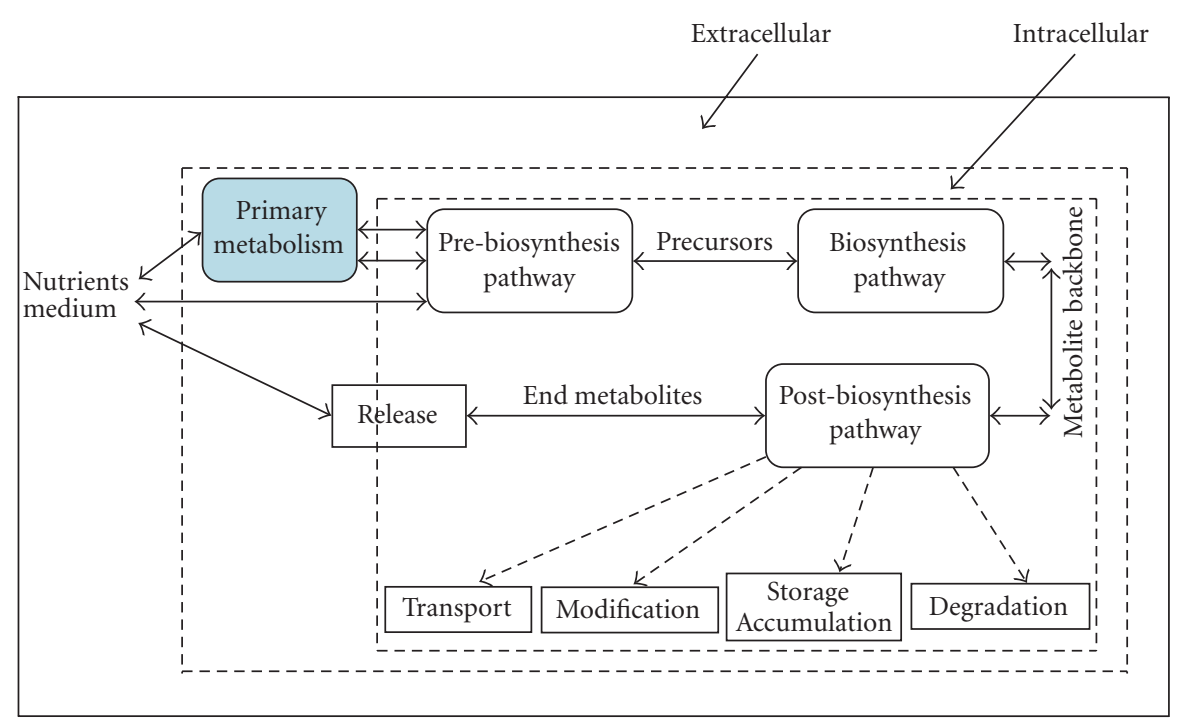

FIGURE 3. Pathway events involved in biosynthesis of a metabolite in plant cells: primary metabolism and secondary metabolism (pre-biosynthetic, biosynthetic, and post-biosynthetic pathways).

combination with a GC-MS-SIM alkaloid analysis, the simultaneous detection of the genetic onset of various secondary metabolic pathways and genetic reprogramming of primary metabolism to sustain secondary metabolism was achieved in a jasmonate-elicited BY-2 tobacco cell culture. The advantage of this approach is the capability of detecting the vast majority of transcripts without any prior sequence knowledge.

Secondly, genome-wide analysis is essential to understand the interactions between the biosynthetic pathways of targeted metabolites and their linked pathways (complimentary and competing pathways). The regulation and manipulation of complimentary pathways provides the means to sustain the targeted pathways, while the blocking of competing pathways is critical for redirecting metabolic flux. One example of these strategies was illustrated by the combination of activation tagging mutagenesis with high-throughput screening for biological active metabolites, which enabled the isolation of genetic material relevant to the synthesis of specific natural products [13]. This approach involved the generation of a callus library via plant cell mutagenesis prepared by activation T-DNA tagging [15], the development of a high-throughput screen assay for the targeted metabolites, and the functional genomics analysis of the overexpressing cell lines. The strategy has the advantage that no prior knowledge of the metabolic pathway is required-only a method of screening for the metabolite products.

Thirdly, the primary metabolism to sustain the respective secondary metabolism has to be characterized. The responses of plant secondary metabolism to any genetic/metabolic manipulation will largely depend on the cell physiological and nutritional state, as an indicator of primary metabolism. The empirical approach optimizes the growth environment with the cells as a black box, thereby bypassing the cell physiological state as an intermediate control objective. In a rational approach, this limitation must be addressed through explicit monitoring and control of cellular physiology. Konstantinov [16] has proposed a generic methodology for the design of systems capable of performing these advanced monitoring and control functions. The physiological state was quantified by a vector composed of several process variables that were selected among different classes, including specific metabolic rates, metabolic rate ratios, degrees of limitation, and others. This was demonstrated using plant cell culture of Perilla frutescens for anthocyanin production as an example. Lamboursain and Jolicoeur [17] highlighted the strong influence of plant cell nutritional status on cell growth and secondary metabolite production capacity of the cells. They defined the nutritional status as the intraand extracellular concentrations in nutrients, total nitrogen, phosphate, and carbohydrate. The lack of information and control of cell nutritional status may be responsible for the poor reproducibility of plant cell bioprocesses.

Finally, the post-biosynthetic events will need to be characterized and targeted for engineering manipulation $[2,6]$. As illustrated in Figure 3, secondary metabolism may include the pre-biosynthetic pathways to produce the precursors, the biosynthetic pathways to produce the core metabolite structures, and the post-biosynthetic events. These post-biosynthetic events-the chemical and enzymatic modifications, transport, storage/secretion, and catabolism/degradation-have been largely unexplored in the past. Zhang et al [2] have demonstrated the significance in characterization and manipulation of these post-biosynthetic events in stretching the biosynthetic capability of plant cells. 


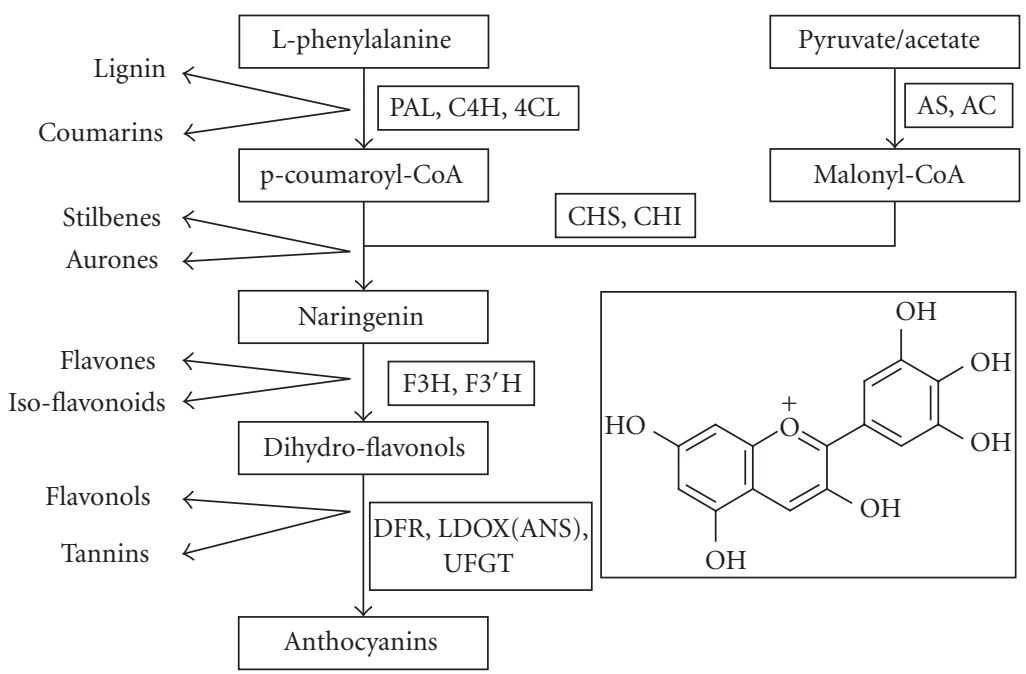

FIGURE 4. Schematic of anthocyanin biosynthetic pathways and the key enzymes involved. Branched pathways leading to other metabolites are also indicated. Phenylalanine ammonia lyase (PAL); cinnamate-4-hydroxylase $(\mathrm{C} 4 \mathrm{H})$; 4-coumarate-CoA ligase (4CL); acetyl-CoA synthetase (AS); acetyl-CoA carboxylase (AC); chalcone synthase (CHS); chalcone isomerase (CHI); flavanone 3 $\beta$ hydroxylase $(\mathrm{F} 3 \mathrm{H})$; flavonoid 3'-hydroxylase $\left(\mathrm{F}^{\prime} \mathrm{H}\right)$; dihydroflavonol 4-reductase (DFR); leucoanthocyanidin dioxygenase (LDOX (ANS)); UDP-glucose:cyanidin 3-O-glucosyltransferase (UFGT).

\section{RATIONAL APPROACH: ANTHOCYANIN AS A CASE STUDY}

In our laboratory, we are interested in the fundamental understanding of the biosynthetic pathways and regulation of secondary metabolism in plant cell cultures with the ultimate goal of rational development of commercial plant cell bioprocesses. The establishment of a rational approach is an ongoing effort toward an advanced knowledge of biosynthesis and post-biosynthesis pathways of anthocyanins in $V$ vinifera cell culture as a model system from genetic to metabolite level $[2,3,4,5,6,7]$.

Anthocyanins, responsible for various attractive colors in plants, are becoming an important alternative to many synthetic colorants and have potential applications in nutraceutical developments [9]. There is a great deal of information available on the anthocyanin biosynthetic pathway as shown in Figure 4. As discussed above, our approach started with the establishment of integrated postgenomic tools to characterize anthocyanin metabolism at transcriptional, enzymatic, and metabolic levels. As the stilbene pathway competes directly for the precursors for anthocyanin biosynthesis, characterization of the stilbene biosynthetic pathway was carried out concurrently. In addition, anthocyanin transport and storage were initially targeted for the elucidation of post-biosynthetic events.

\section{Transcriptional, enzymatic, and metabolic characterization of anthocyanin pathways}

Using techniques such as precursor feeding, elicitation, metabolic inhibition, redirected transport, and analysis of strains, the dynamic profiles of mRNA expres- sion, enzyme activities, and anthocyanin metabolites of the biosynthetic pathways in $V$ vinifera cell culture were characterized. One example was the functional analysis of $V$ vinifera cell cultures that were elicited with jasmonic acid, light, and sucrose alone and in combination $[3,4]$. All these single conditions enhanced anthocyanin production and exhibited a synergistic improvement when combined $[3,4]$. Early transcriptional studies were done by Northern blotting, and later quantitative RT-PCR, with a sample of results shown in Figure 5. Results indicated a strong correlation between transcriptional expression and improved anthocyanin biosynthesis and a role of jasmonic acid in upregulating DFR [4].

Metabolic profiling was also carried out substantively for these conditions. Results implicated the competition between anthocyanin and stilbene pathways, and the importance of methylated and acylated anthocyanin species in enhanced production [5]. Full characterization is in progress following the completion of the methodology development.

\section{Characterization of anthocyanin post-biosynthetic events}

Anthocyanins are synthesized in the cytoplasm and transported into the vacuole where they bind with a protein matrix and form anthocyanic vacuolar inclusions (AVIs) (Figure 6a) [6]. AVIs were considered to be the storage sites of anthocyanins. In recognition that the post-biosynthetic steps may play equally crucial roles in its yield improvement, we have been investigating the characteristics and roles of glutathione S-transferases (GSTs) and AVIs in anthocyanin transport and storage, respectively, in grape cells. We have isolated the AVIs and are 


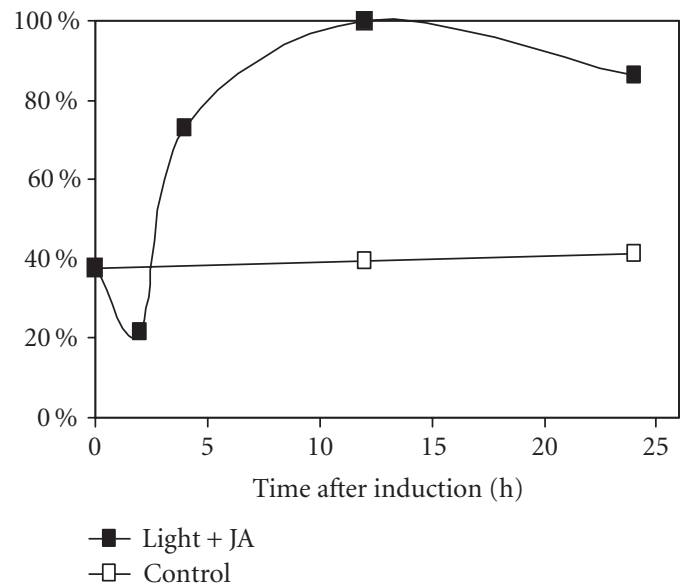

(a)

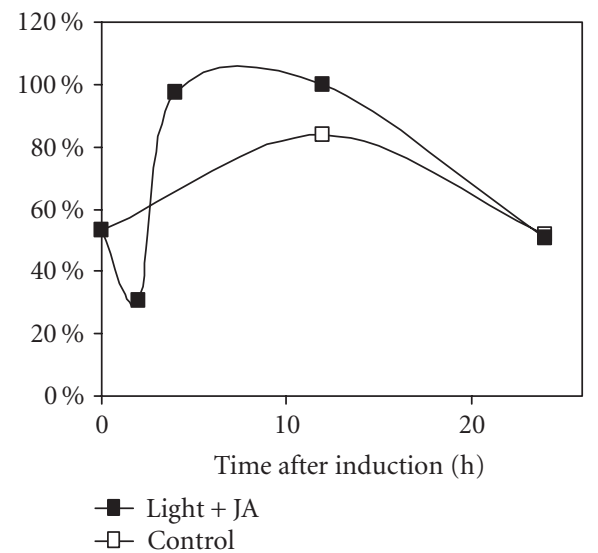

(c)

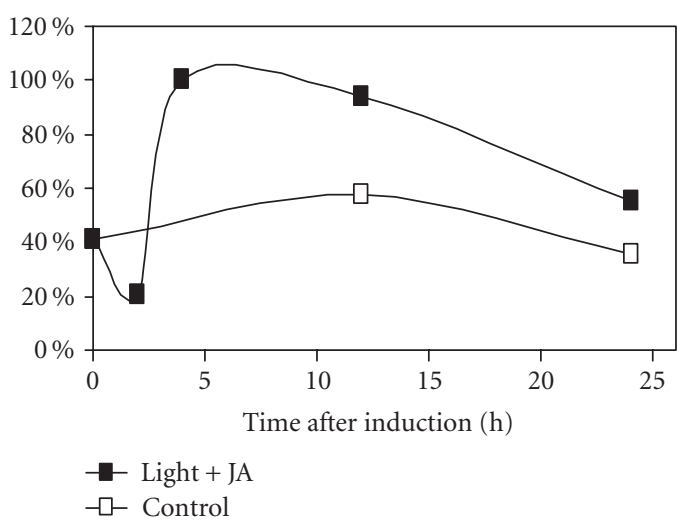

(e)

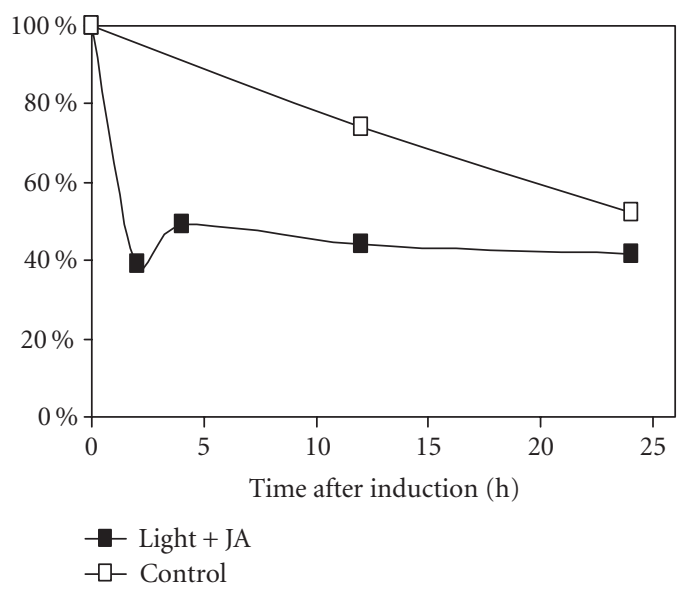

(b)

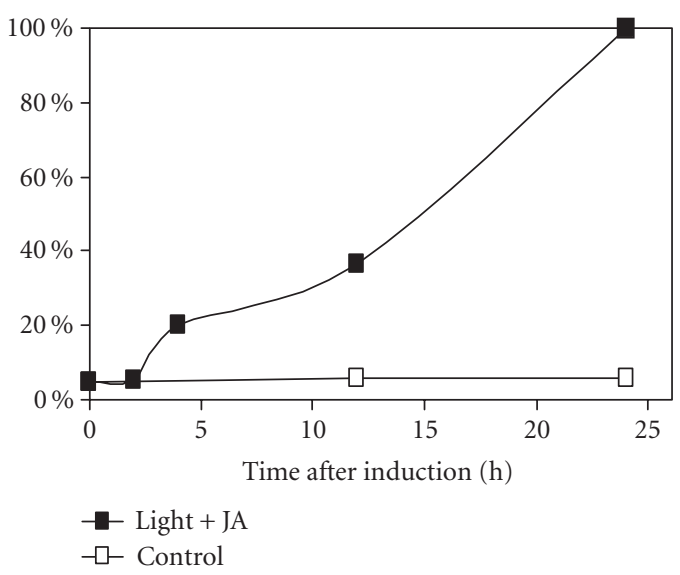

(d)

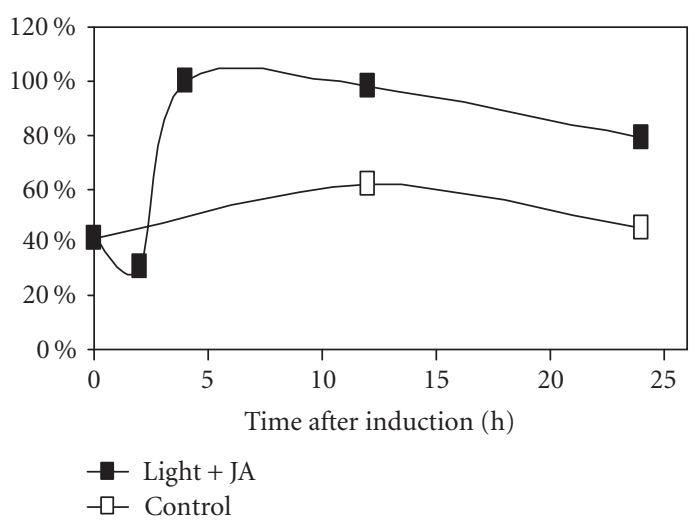

(f)

FIGURE 5. Quantification of mRNA level for six anthocyanin biosynthesis genes in $V$ vinifera cell culture grown under darkness (control), and under light of 8000 Lux with JA elicitation on day 0. The RNA was probed with $V$ vinifera cDNA clones for (a) CHS, (b) CHI, (c) F3H, (d) DFR, (e) LDOX, and (f) UFGT. Ribosomal RNA was used as an internal control. Images of Northern blot were visualized by Phosphorimager, corrected with respective rRNA, and quantified using ImageQuant software.

characterizing them using an integrated post-genomic approach as mentioned above. Initial results indicated AVIs may be composed of several protein species and have the selectivity for acylated anthocyanins (Figures $6 \mathrm{~b}$ and $6 \mathrm{c}$ ). It is expected that these studies will provide additional targets for rational metabolic engineering [2]. 


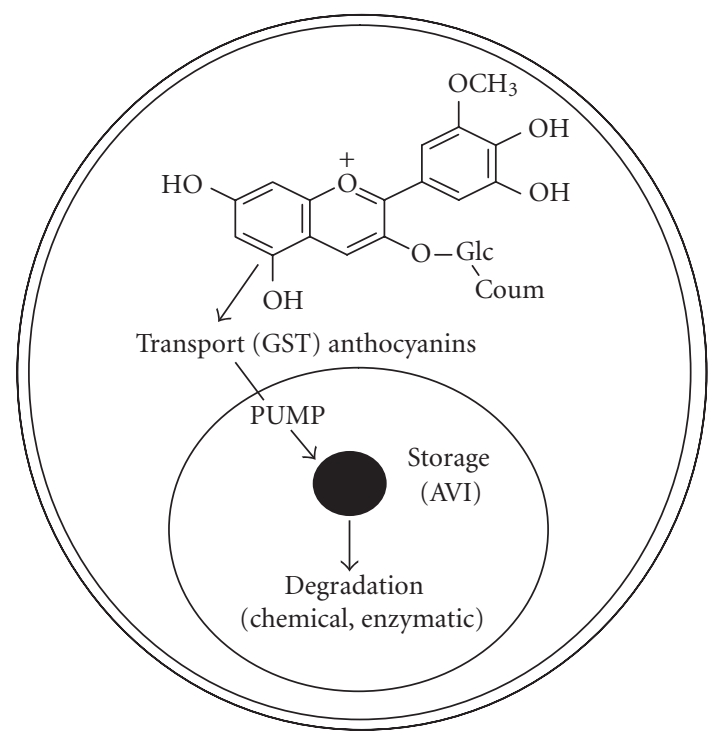

(a)

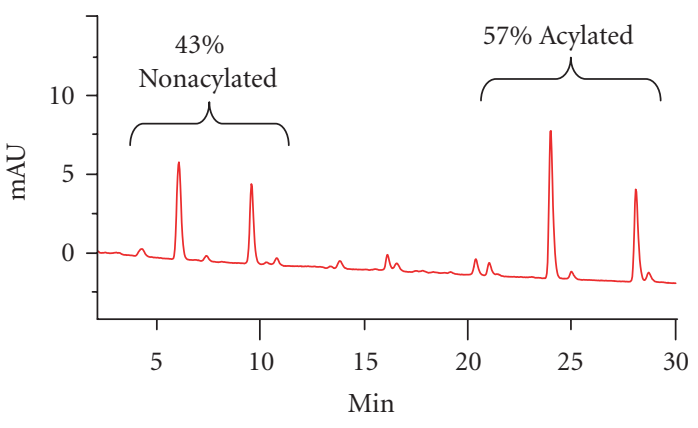

(b)

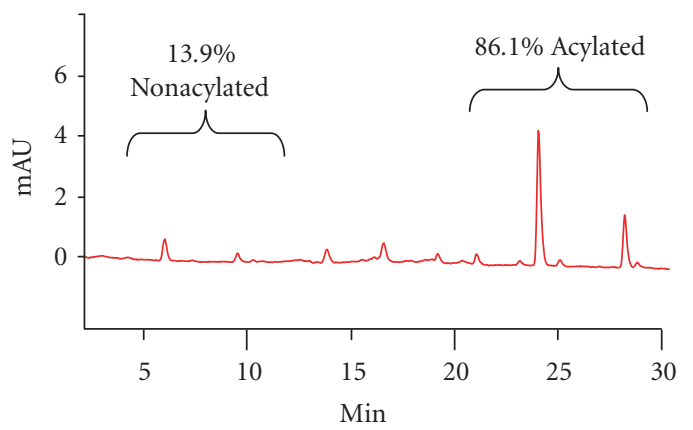

(c)

FIGURE 6. (a) A schematic of anthocyanin post-biosynthetic events. (b) HPLC profiles of whole cell. (c) HPLC profile of AVI extracts of the grape cell line at $520 \mathrm{~nm}$. Nonacylated and acylated (p-coumaroylated) species are grouped and mean percentages of total peak area are shown $(n=4) . \mathrm{mAU}=$ milli absorbance units.

\section{ACKNOWLEDGMENT}

This research was financially supported by the CRC for Bioproducts, Australia, and the National Natural Science Foundation of China (No 20176058).

\section{REFERENCES}

[1] Hadacek F. Secondary metabolites as plant traits: current assessment and future perspectives. CRC Crit Rev Plant Sci. 2002;21(4):273-322.

[2] Zhang W, Curtin C, Franco C. Towards manipulation of post-biosynthetic events in secondary metabolism of plant cell cultures. Enzyme Microb Technol. 2002;30(6):688-696.

[3] Zhang W, Curtin C, Kikuchi M, Franco C. Integration of jasmonic acid and light irradiation for enhancement of anthocyanin biosynthesis in Vitis vinifera suspension cultures. Plant Sci. 2002;162(3): 459-468.

[4] Kikuchi M. Transcriptional regulation of enzymes involved in anthocyanin biosynthesis in Vitis vinifera cell suspension culture. Master's thesis, Flinders University, Adelaide, South Australia, Australia, 2001.

[5] Curtin C, Zhang W, Franco C. Manipulating anthocyanin composition in Vitis vinifera suspension cultures by elicitation with jasmonic acid and light irradiation. Biotechnol Lett. 2003; 25(14):1131-1135.

[6] Conn S, Zhang W, Franco C. Anthocyanic vacuolar inclusions (AVIs) selectively bind acylated anthocyanins in Vitis vinifera L (grapevine) suspension culture. Biotechnol Lett. 2003;25(11):835-839.

[7] Springob K, Nakajima J, Yamazaki M, Saito K. Recent advances in the biosynthesis and accumulation of anthocyanins. Nat Prod Rep. 2003;20(3):288-303.

[8] Roberts SC, Shuler ML. Large-scale plant cell culture. Curr Opin Biotechnol. 1997;8(2):154-159.

[9] Zhang W, Furusaki S. Production of anthocyanins by plant cell cultures. Biotechnol Bioprocess Eng. 1999;4 (4):231-252.

[10] Dörnenburg H, Knorr D. Strategies for the improvement of secondary metabolite production in plant cell cultures. Enzyme Microb Technol. 1995;17(8): 674-684.

[11] Oksman-Caldentey K, Häkkinen S, Goossens A, et al. Secondary metabolites in the post-genomic era. In: Vasil IK, ed. Plant Biotechnology 2002 and Beyond. Dordrecht: Kluwer Academic Publishers; 2003:465468.

[12] Willmitzer L. About straight lines and complex crossroads: metabolism is a network. In: Vasil IK, ed. Plant Biotechnology 2002 and Beyond. Dordrecht: Kluwer Academic Publishers; 2003:81.

[13] Falcone DL, Rogers DT, Yun KY, et al. A functional genomics strategy to identify genes that regulate the production of biologically active metabolites in plants. In: Vasil IK, ed. Plant Biotechnology 2002 
and Beyond. Dordrecht: Kluwer Academic Publishers; 2003:469-472.

[14] Goossens A, Hǎkkinen ST, Laakso I, et al. A functional genomics approach toward the understanding of secondary metabolism in plant cells. Proc Natl Acad Sci USA. 2003;100(14):8595-8600.

[15] Fritze K, Walden R. Gene activation by T-DNA tagging. Methods Mol Biol. 1995;44:281-294.

[16] Konstantinov KB. Monitoring and control of the physiological state of cell cultures. Biotechnol Bioeng. 1996;52(2):271-289.

[17] Lamboursain L, Jolicoeur M. In vitro production of secondary metabolites by cultivated plant cells: the crucial role of the cell nutritional status. In: Vasil IK, ed. Plant Biotechnology 2002 and Beyond. Dordrecht: Kluwer Academic Publishers; 2003:491-495.

* Corresponding author.

E-mail: wei.zhang@flinders.edu.au

Fax: +61 88204 4101; Tel: +61 882045053 

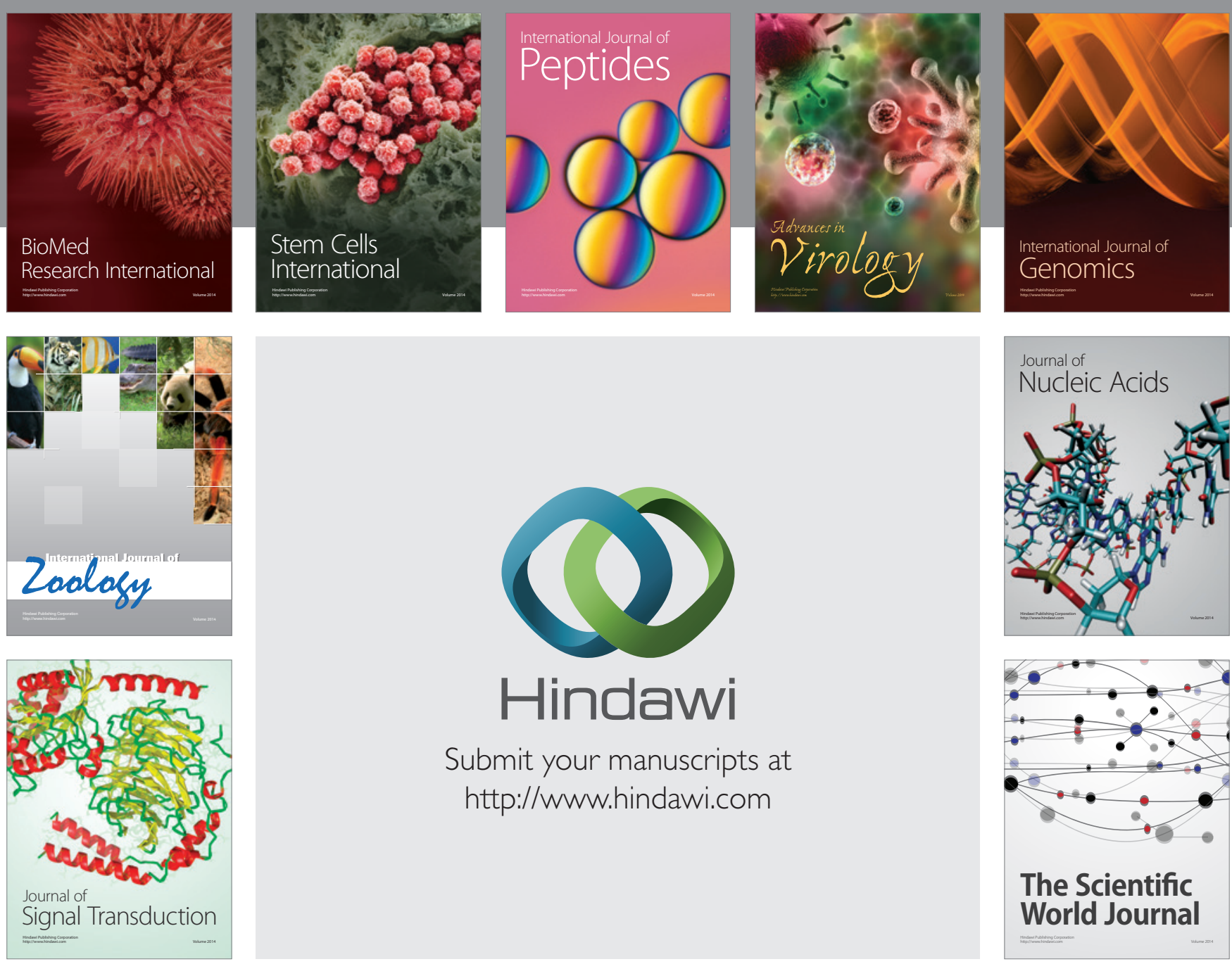

Submit your manuscripts at

http://www.hindawi.com
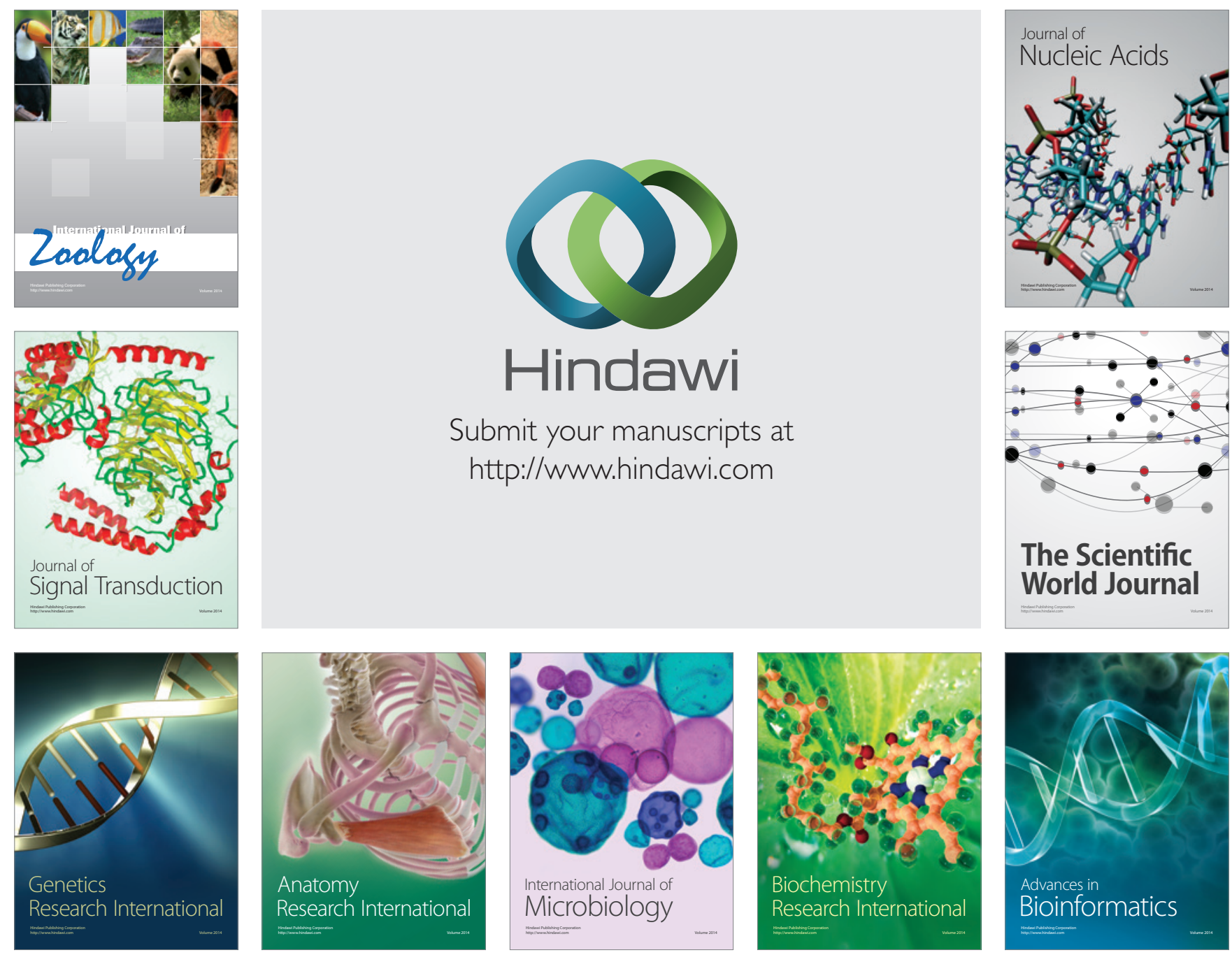

The Scientific World Journal
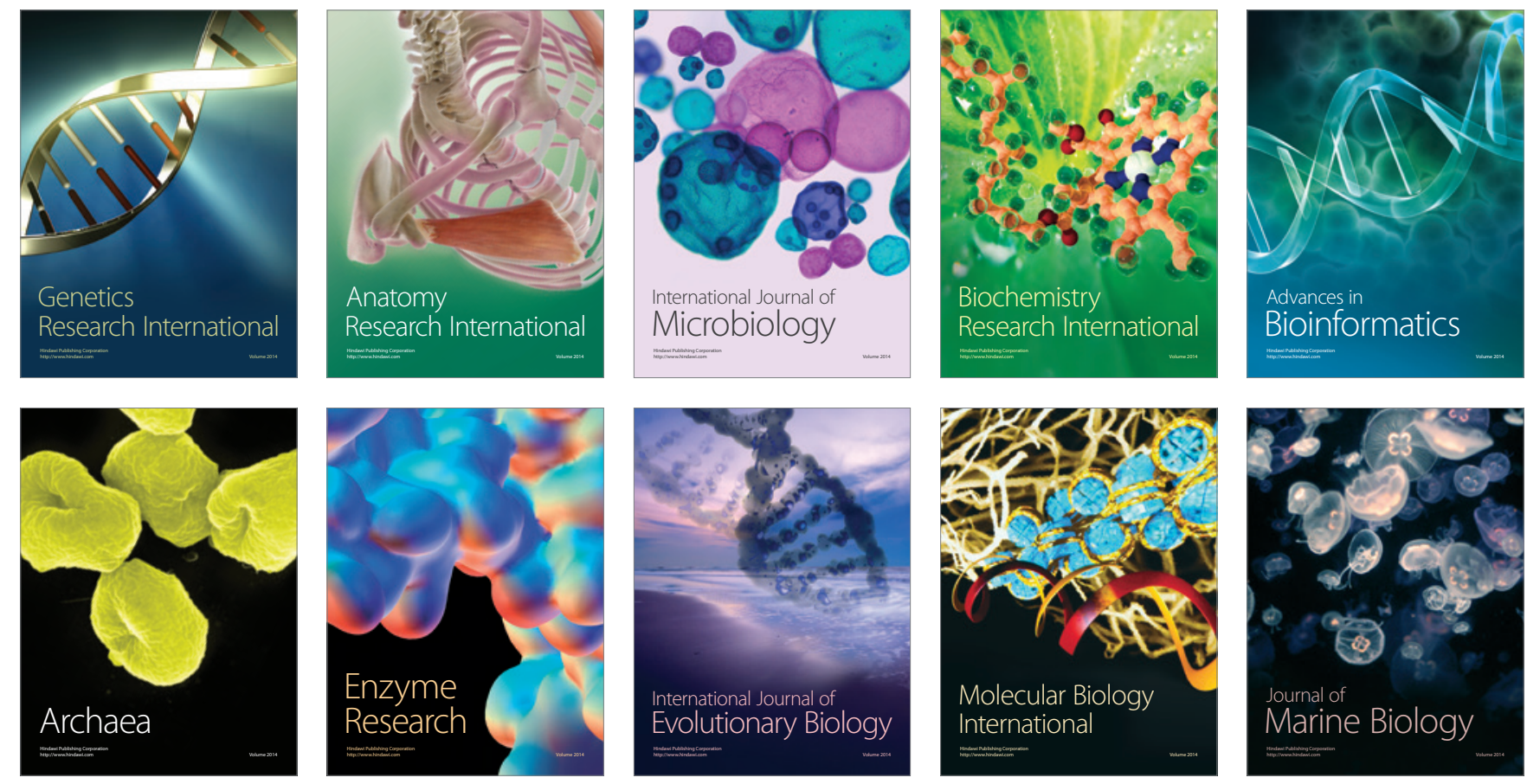